\title{
Article
}

\section{On divisible and pure multigroups and their properties}

\author{
P. A. Ejegwa ${ }^{1, *}$ and M. A. Ibrahim ${ }^{2}$ \\ 1 Department of Mathematics, University of Agriculture, Makurdi, Nigeria. \\ 2 Department of Mathematics, Ahmadu Bello University, Zaria, Nigeria. \\ * Correspondence: ejegwa.augustine@uam.edu.ng
}

Received: 18 June 2020; Accepted: 8 October 2020; Published: 23 October 2020.

\begin{abstract}
The theory of multigroups is a generalized group's theoretic notions in multiset framework. Although myriad of researches have been done in multigroup theory, but some group's analogue concepts have not been investigated in multigroup setting. In this paper we propose the notions of divisible and pure multigroups and characterize some of their properties. It is established that the image and preimage of homomorphism of divisible and pure multigroups are divisible and pure multigroups. The nexus between divisible and pure multigroups and that of divisible and pure groups are instituted using the concept of cuts of multigroups.
\end{abstract}

Keywords: Multiset, multigroup, divisible multigroup, pure multigroup, divisible group, pure group.

MSC: 03E72, 06D72, 11E57, 19A22.

\section{Introduction}

$\mathbf{T}$ he term multisets as buttressed by Knuth [1], was first recommended by N. G. de Bruijn (cf. [2]) in a communication to D. E. Knuth, as a generalization of classical sets, by violating the principle of distinct collection of elements in set theory. Both the theory and applications of multiset have been extensively studied in [3-8]. In a way to apply group theory to multisets, Nazmul et al., [9] proposed the concept of multigroups in multisets framework and presented a number of results, just as group theory stem out of set theory. A comprehensive account on the concept of multigroups has been carried out, and it has been established that multigroup via multiset is a generalization of group theory [10].

Several researches have emerged on the concept of multigroup theory. Some results on multigroups which cut across some homomorphic properties were explored in [11,12]. The notions of upper and lower cuts of multigroups were introduced and discussed in details with some number of results in [13], and the notions were extended to homomorphism in [14]. Some group's analogous concepts like normal subgroups, characteristic subgroups, direct product, cosets, factor groups and group actions, etc. have been established in multigroup context [15-27]. The idea of commutators in multigroup context has been studied in [28]. The notion of ring structure from multiset context has been proposed and some related results deduced [29].

Though numerous constructs in group theory have been established in multigroup structure, some constructs have not been considered in multigroup context. The notions of divisible and pure groups have been hitherto studied in other non-classical groups like fuzzy groups and intuitionistic fuzzy groups, respectively [30-32]. This paper attempts to strengthen multigroup theory by characterizing the constructs of divisible and pure multigroups which are the applications of divisible and pure groups in multisets. We first defined a special multisets of a group to boost the introduction of divisible and pure multigroups. We study the homomorphic image and preimage of divisible and pure multigroups and found that they are divisible and pure multigroups. The correlation between divisible and pure multigroups and that of divisible and pure groups are established using the idea of multigroup cuts. The rest of the paper is outlined as follows; Section 2 presents the ideas of multisets, multigroups and some helpful existing results. Section 3 discusses and characterizes divisible multigroups. Finally, Section 4 gives a precise conclusion with area of future research.

\section{Preliminaries}

Throughout this paper $X$ denotes non-empty set and $G$ denotes an additive group with identity element 0. 
Definition 1. [8] Let $X$ be a set. A multiset $A$ over $X$ is just a pair $\left\langle X, C_{A}\right\rangle$, where

$$
C_{A}: X \rightarrow \mathcal{N}=\{0,1,2, \ldots\}
$$

is a function, such that for $x \in X$ implies $A(x)$ is a cardinal and $A(x)=C_{A}(x)>0$, where $C_{A}(x)$ denoted the number of times an object $x$ occur in $A$. Whenever $C_{A}(x)=0$, implies $x \notin X$. The set $X$ is called the ground or generic set of the class of all multisets containing objects from $X$.

Definition 2. [7] Let $X$ be the set from which multisets are constructed. The multiset $X^{n}$ is the set of all multisets of $X$ such that no element occurs more than $n$ times. Likewise, the multiset $X^{\infty}$ is the set of all multisets of $X$ such that there is no limit on the number of occurrences of an element.

Definition 3. [7] Let $A, B$ be multisets of $X$. Then

(i) $A=B \Longleftrightarrow C_{A}(x)=C_{B}(x) \forall x \in X$,

(ii) $A \subseteq B \Longleftrightarrow C_{A}(x) \leq C_{B}(x) \forall x \in X$,

(iii) $A \cap B \Longrightarrow C_{A \cap B}(x)=C_{A}(x) \wedge C_{B}(x) \forall x \in X$,

(iv) $A \cup B \Longrightarrow C_{A \cup B}(x)=C_{A}(x) \vee C_{B}(x) \forall x \in X$,

(v) $A \oplus B \Longrightarrow C_{A \oplus B}(x)=C_{A}(x) \oplus C_{B}(x) \forall x \in X$,

where $\wedge$ and $\vee$ denote minimum and maximum respectively.

Definition 4. [9] Suppose $\left\{A_{i}\right\}_{i \in I}$ is an arbitrary family of multisets of $X$. Then $\bigcap_{i \in I} A_{i}=\bigwedge_{i \in I} C_{A_{i}}(x) \forall x \in X$ and $\bigcup_{i \in I} A_{i}=\bigvee_{i \in I} C_{A_{i}}(x) \forall x \in X$.

Definition 5. [9] A multiset $A$ of $G$ is called a multigroup if

(i) $C_{A}(x+y) \geq C_{A}(x) \wedge C_{A}(y) \forall x, y \in G$,

(ii) $C_{A}(-x)=C_{A}(x) \forall x \in G$.

From (i) and (ii), we have $C_{A}(x-y) \geq C_{A}(x) \wedge C_{A}(y) \forall x, y \in G$. In fact, in a multigroup $A$ of $G, C_{A}(0) \geq$ $C_{A}(x) \forall x \in G$.

Definition 6. [10] A multigroup $A$ of $G$ is said to be constant or has a constant count function if $C_{A}(x)=C_{A}(y)$ $\forall x, y \in G$.

Definition 7. [10] A multigroup $A$ of $G$ is said to be commutative if and only if

$$
C_{A}(x+y)=C_{A}(y+x) \forall x, y \in G .
$$

Proposition 1. [9] If $A$ and $B$ are multigroups of $G$, then (i) $A \cap B$ and $A \oplus B$ are multigroups of $G$, (ii) $A \cup B$ is a multigroup of $G$ provided $A \subseteq B$.

Theorem 1. [13] Let $A$ be a multigroup of $G$. Then, the sets $A_{n}$ and $A^{n}$ defined by

$$
A_{n}=\left\{x \in G \mid C_{A}(x) \geq n\right\}
$$

and

$$
A^{n}=\left\{x \in G \mid C_{A}(x) \leq n\right\}
$$

where $n \in \mathbb{N}$, are subgroups of $G$ for $n \leq C_{A}(0)$ and $n \geq C_{A}(0)$, respectively.

Theorem 2. [22] Let $A$ be a multiset of $G$ and $n \in \mathbb{N}$.

(i) If every $A_{n}$ for $n \leq C_{A}(0)$ is a subgroup of $G$, then $A$ is a multigroup of $G$.

(ii) If every $A^{n}$ for $n \geq C_{A}(0)$ is a subgroup of $G$, then $A$ is a multigroup of $G$.

Definition 8. [12] Let $f: G \rightarrow G^{\prime}$ be a homomorphism of groups. Suppose $A$ and $B$ are multigroups of $G$ and $G^{\prime}$, respectively. Then, $f$ induces a homomorphism from $A$ to $B$ which satisfies

(i) $C_{A}\left(f^{-1}\left(y_{1} y_{2}\right)\right) \geq C_{A}\left(f^{-1}\left(y_{1}\right)\right) \wedge C_{A}\left(f^{-1}\left(y_{2}\right)\right) \forall y_{1}, y_{2} \in G^{\prime}$, 
(ii) $C_{B}\left(f\left(x_{1} x_{2}\right)\right) \geq C_{B}\left(f\left(x_{1}\right)\right) \wedge C_{B}\left(f\left(x_{2}\right)\right) \forall x_{1}, x_{2} \in G$,

where

(i) the image of $A$ under $f$, denoted by $f(A)$, is a multiset of $G^{\prime}$ defined by

$$
C_{f(A)}(y)= \begin{cases}\bigvee_{x \in f^{-1}(y)} C_{A}(x), & f^{-1}(y) \neq \varnothing \\ 0, & \text { otherwise }\end{cases}
$$

for each $y \in G^{\prime}$.

(ii) the preimage of $B$ under $f$, denoted by $f^{-1}(B)$, is a multiset of $G$ defined by

$$
C_{f^{-1}(B)}(x)=C_{B}(f(x)) \forall x \in G
$$

Proposition 2. [12] Let $f: G \rightarrow G^{\prime}$ be a homomorphism of groups, $A$ and $B$ be multigroups of $G$ and $G^{\prime}$, respectively. Then $f(A)$ is multigroup of $G^{\prime}$ and if $f$ is an isomorphism, then $f^{-1}(B)$ is a multigroup of $G$.

\section{Divisible multigroups and pure multigroups}

This section introduces the concepts of divisible and pure multigroups and presents some of their properties.

\subsection{Some properties of divisible multigroups}

Recall that an abelian group $G$ is divisible if for every positive integer $n$ and any $x \in G, \exists y \in G$ such that $n y=x$. Equivalently, an abelian group $G$ is divisible if and only if $n G=G$, where $n$ is any positive integer and $n G=\{n x \mid x \in G\}$.

Definition 9. Let $A$ be a multiset of $G$. Then, the set $n A$, where $n$ is a positive integer is defined as

$$
C_{n A}(x)= \begin{cases}\bigvee_{x=n y} C_{A}(y), & x \in n G \\ 0, & \text { otherwise }\end{cases}
$$

Proposition 3. Suppose $A$ and $B$ are multisets of $G$, then

(i) $A \subseteq B \Longrightarrow n A \subseteq n B$,

(ii) $n(A \cap B)=n A \cap n B$,

(iii) $n(A \cup B)=n A \cup n B$,

(iv) $n(A \oplus B)=n A \oplus n B$.

Proof. The proof of (i) is trivial. Now, we prove (ii) as follows. Suppose $x \notin n G$, then $C_{n(A \cap B)}(x)=0=$ $C_{n A \cap n B}(x)$. Again, assume $x \in n G$, then we have

$$
\begin{aligned}
C_{n A \cap n B}(x) & =C_{n A}(x) \wedge C_{n B}(x) \\
& =\bigvee_{x=n y} C_{A}(y) \wedge \bigvee_{x=n y} C_{B}(y) \\
& =\bigvee_{x=n y}\left[C_{A}(y) \wedge C_{B}(y)\right] \\
& =\bigvee_{x=n y} C_{A \cap B}(y) \\
& =C_{n(A \cap B)}(x) .
\end{aligned}
$$

Hence, $n(A \cap B)=n A \cap n B$. The proofs of (iii) and (iv) follow directly from Definitions 3, 9 and (ii).

Theorem 3. Suppose $f: G \rightarrow G^{\prime}$ is a homomorphism such that $A$ is multiset of $G$. Then for any $n \in \mathbb{N}, f(n A)=$ $n f(A)$. 
Proof. Suppose $x \notin n G$, then $C_{f(n A)}(x)=0=C_{n f(A)}(x)$. Again, suppose we have $x \in n G$. If $z \in G^{\prime}$ and $z=f(x)$, then

$$
\begin{aligned}
C_{f(n A)}(z) & =\bigvee_{z=f(x)} C_{n A}(x)=\bigvee_{z=f(x)} \bigvee_{x=n w} C_{A}(w) \\
& =\bigvee_{z=f(x), x=n w} C_{A}(w)=\bigvee_{z=f(n w)} C_{A}(w) \\
& =\bigvee_{z=n f(w)} C_{A}(w)=\bigvee_{z=n y} \bigvee_{y=f(w)} C_{A}(w) \\
& =\bigvee_{z=f(n w)} C_{f(A)}(y)=C_{n f(A)}(z) .
\end{aligned}
$$

Hence, $f(n A)=n f(A)$.

Theorem 4. Let $f$ be a homomorphism from $G$ to $G^{\prime}$ such that $B$ is a multiset of $G^{\prime}$. Then for any $n \in \mathbb{N}, n f^{-1}(B) \subseteq$ $f^{-1}(n B)$. Moreover if $f$ is an isomorphism, $n f^{-1}(B)=f^{-1}(n B)$.

Proof. Let $x \in G$. If $x \notin n G$, then $C_{n f^{-1}(B)}(x)=0 \leq C_{f^{-1}(n B)}(x)$. If $x \in n G$, then $f(x) \in n G^{\prime}$. Thus

$$
\begin{aligned}
C_{n f^{-1}(B)}(x) & =\bigvee_{x=n w} C_{f^{-1}(B)}(w)=\bigvee_{x=n w} C_{B}(f(w)) \\
& \leq \bigvee_{f(x)=f(n w)} C_{B}(f(w)) \leq \bigvee_{f(x)=n y} C_{B}(y) \\
& =C_{n B}(n y)=C_{n B}(f(x)) \\
& =C_{f^{-1} B}(x) .
\end{aligned}
$$

Hence, $n f^{-1}(B) \subseteq f^{-1}(n B)$. Suppose $f$ is an isomorphism, then by using the argument in Theorem 3, we have

$$
\begin{aligned}
C_{f^{-1}(n B)}(x) & =C_{f^{-1}\left(n\left(f\left(f^{-1}(B)\right)\right)\right)}(x)=C_{f^{-1}\left(f\left(n\left(f^{-1}(B)\right)\right)\right)}(x) \\
& \leq C_{n\left(f^{-1}(B)\right)}(x),
\end{aligned}
$$

so $f^{-1}(n B) \subseteq n f^{-1}(B)$. Therefore, $n f^{-1}(B)=f^{-1}(n B)$.

Definition 10. Let $G$ be an abelian group. A multigroup $A$ of $G$ is called divisible if $C_{n A}(x)=C_{A}(x) \forall x \in G$ for every positive integer $n$. Equivalently, if $n A=A$ for every positive integer $n$.

Example 1. A multigroup of (i) an additive group of rational numbers $\mathbb{Q}$ is a divisible multigroup, (ii) a group of complex roots of unity of degrees $p^{k}, k=1, \ldots, n$, where $p$ is a prime number is a divisible multigroup.

Remark 1. (i) Every multigroup of a divisible group is a divisible commutative multigroup. (ii) If $G=\mathbb{Q}$, then every divisible multigroup of $G$ has a constant count function over $\mathbb{Q}-\{0\}$.

Proposition 4. Suppose $f: G \rightarrow G^{\prime}$ is a homomorphism of groups, and $A$ be a divisible multigroup of $G$. Then, the homomorphic image of $A$ is a divisible multigroup of $G^{\prime}$.

Proof. From Theorem 2, we see that $f(A)$ is a multigroup of $G^{\prime}$. Thus, we have $n f(A)=f(n A)=f(A)$, for every $n \in \mathbb{N}$ (Theorem 3). Hence, $f(A)$ is a divisible multigroup of $G$.

Proposition 5. Let $f: G \rightarrow G^{\prime}$ be an isomorphism of groups, and $B$ be a divisible multigroup of $G^{\prime}$. Then, the homomorphic preimage of $B$ is a divisible multigroup of $G$.

Proof. By synthesizing Theorems 2 and 4 , it follows that $f^{-1}(B)$ is a divisible multigroup of $G$.

Definition 11. A multigroup A of $G$ is called p-divisible if and only if $C_{p^{k} A}(x)=C_{A}(x) \forall x \in G$ where $k \in \mathbb{N}$ and $p$ is a prime. 
Proposition 6. Let $A$ be a multigroup of $G$. Then $A$ is divisible if and only if it is p-divisible for every prime $p$.

Proof. Suppose that $A$ is divisible multigroup of $G$. Certainly, it is p-divisible since $p^{k} \in \mathbb{N}$.

Conversely, assume $A$ is a p-divisible multigroup of $G$ for every prime $p$. Then for every $n \in \mathbb{N}$, we get $n=p_{1} p_{2} \ldots p_{m}$ where $p_{i}($ for $i=1, \ldots, m)$ is prime. Thus, $n A=\left(p_{1} p_{2} \ldots p_{m}\right) A=\beta A=A$ since $n=\beta$. So, $A$ is a divisible multigroup of $G$.

Theorem 5. Let A be a divisible multigroup of $G$. Then, the following are divisible subgroups of $G$ :

(i) $A_{n}, n \in \mathbb{N}$ for $n \leq C_{A}(0)$.

(ii) $A^{n}, n \in \mathbb{N}$ for $n \geq C_{A}(0)$.

Proof. (i) By Theorem $1, A_{n}$ is a subgroup of $G$. Let $x \in A_{n}$ and $n \in \mathbb{N}$. Since $\bigvee_{n y=x} C_{A}(y)=C_{A}(x) \geq n$, then it follows that $y \in A_{n}$ with $n y=x$. Hence, $A_{n}$ is a divisible subgroup of $G$.

(ii) Similarly, $A^{n}$ is a subgroup of $G$ by Theorem 1 . If $x \in A^{n}$ and $n \in \mathbb{N}$. Then, since $\bigvee_{n y=x} C_{A}(y)=C_{A}(x) \leq$ $n$, it follows that $y \in A^{n}$ such that $n y=x$, and the result follows.

Theorem 6. Let $A$ be a multiset of $G$ bounded by $G^{n}$ such that $C_{A}(0)=n$ and every $A_{n}, n \in \mathbb{N}$, is a divisible subgroup of $G$. Then $A$ is a divisible multigroup of $G$.

Proof. From the given hypotheses, it follows that $A$ is a multigroup of $G$ by Theorem 2. Let $x \in G$ and $C_{A}(x)=n$. Since $A_{n}$ is a divisible subgroup of $G$ for every $n \in \mathbb{N}, \exists y \in A_{n}$ such that $n y=x$, hence $C_{A}(y) \geq C_{A}(x)=n$. But $C_{A}(y) \leq C_{A}(x)$ since $A$ is a multigroup of $G$. Hence, $\bigvee_{n y=x} C_{A}(y)=C_{n A}(x)$, for $x \in n G$ and so, $n A=A$.

Corollary 7. If $A$ is a multiset of $G$ bounded by $G^{n}$ such that $C_{A}(0)=n$ and every $A^{n}, n \in \mathbb{N}$, is a divisible subgroup of $G$. Then $A$ is a divisible multigroup of $G$.

Proof. Combining Theorems 2 and 6, the result holds.

Theorem 8. Suppose $\left\{A_{i}\right\}_{i \in I}$ is a family of divisible multigroups of $G$. Then $\bigcap_{i \in I} A_{i}$ is a divisible multigroup of $G$.

Proof. By Proposition $1, \bigcap_{i \in I} A_{i}$ is a multigroup of $G$. Assume that every $A_{i}$ is divisible, then for $x \in n G$ we get

$$
\begin{aligned}
C_{n\left(\cap_{i \in I} A_{i}\right)}(x) & =\bigvee_{x=n y} \bigwedge_{i \in I} C_{A_{i}}(y)=\bigwedge_{i \in I} \bigvee_{x=n y} C_{A_{i}}(y) \\
& =\bigwedge_{i \in I} C_{n A_{i}}(x)=C_{\bigcap_{i \in I}\left(n A_{i}\right)}(x) \\
& =C_{\bigcap_{i \in I} A_{i}}(x) .
\end{aligned}
$$

If $x \notin n G$, then $C_{\bigcap_{i \in I} A_{i}}(x)=0=C_{n\left(\bigcap_{i \in I} A_{i}\right)}(x)$. Hence, $\bigcap_{i \in I} A_{i}$ is divisible.

Theorem 9. Suppose $\left\{A_{i}\right\}_{i \in I}$ is a family of divisible multigroups of $G$. Then

(i) $\bigcup_{i \in I} A_{i}$ is a divisible multigroup of $G$ if $A_{1} \subseteq A_{2} \subseteq \ldots \subseteq A_{n}, i=1, \ldots, n$.

(ii) $\Sigma_{i \in I} A_{i}$ is a divisible multigroup of $G$.

Proof. By using Proposition 1 and following the same logic in Theorem 8, the proofs of (i) and (ii) are established.

Theorem 10. Suppose $A$ is a multigroup of a divisible cyclic group $G$, then $A$ is constant. 
Proof. Let $G=\left\langle a>\right.$ and $x \in G$. Then $x=p a, p \in \mathbb{N}$. Suppose that $C_{A}(p a)>C_{A}((p+1) a)$. Because $G$ is divisible $\exists y \in G$ such that $p y=a$. But also $\exists q \in \mathbb{N}$ such that $y=q a$, and so

$$
\begin{aligned}
C_{A}(p a) & >C_{A}((p+1) a)=C_{A}((p+1) p y) \\
& =C_{A}((p+1) p q a)=C_{A}((p+1) q(p a)) \\
& \geq C_{A}(p a) \wedge C_{A}(p a) \wedge \ldots \wedge C_{A}(p a) \\
& =C_{A}(p a),
\end{aligned}
$$

which is a contradiction.

Also, if $C_{A}((p+1) a)>C_{A}(p a)$. Then $\exists z \in G$ such that $(p+1) z=a, z=r a, r \in \mathbb{N}$ since $G$ is divisible. Thus

$$
\begin{aligned}
C_{A}((p+1) a) & >C_{A}(p a)=C_{A}(p(p+1) r a)=C_{A}(p r(p+1) a) \\
& \geq C_{A}((p+1) a) \wedge C_{A}((p+1) a) \wedge \ldots \wedge C_{A}((p+1) a) \\
& =C_{A}((p+1) a)
\end{aligned}
$$

which is also a contradiction. Hence, $C_{A}((p+1) a)=C_{A}(p a)$. Since $x$ is arbitrary, if $x=a$, then

$$
C_{A}(a)=C_{A}(2 a)=C_{A}(3 a)=\ldots,
$$

and so $A$ is a constant multigroup of a divisible cyclic group $G$.

\subsection{Some properties of pure multigroups}

Recall that a subgroup $H$ of a group $G$ is called pure if $n H=H \cap n G$, for every positive integer $n$. Now, we extend the concept to multigroup of $G$ as follows.

Definition 12. Let $A$ and $B$ be multigroups of $G$ such that $A \subseteq B$. Then $A$ is a pure multigroup of $G$ if $n A=A \cap n B$ for every $n \in \mathbb{N}$. Equivalently, $C_{n A}(x)=C_{A}(x) \wedge C_{n B}(x) \forall x \in G$ for every $n \in \mathbb{N}$.

Remark 2. Suppose $A, B$ and $C$ are multigroups of $G$ such that $A$ and $B$ are contained in $C$. If $A$ and $B$ are pure, then $A \cap B$ and $A \cup B$ are pure multigroups of $G$ since $n(A \cap B)=(A \cap B) \cap n C$ and $n(A \cup B)=(A \cup B) \cap n C$.

Definition 13. Let $A$ and $B$ be multigroups of $G$ such that $A \subseteq B$. Then $A$ is p-pure if $p^{r} A=A \cap p^{r} B$ for every $r \in \mathbb{N}$ and $p$ is prime. Equivalently, if $C_{p^{r} A}(x)=C_{A}(x) \wedge C_{p^{r} B}(x) \forall x \in G$ for every $r \in \mathbb{N}$ and $p$ is prime.

In fact, every p-pure multigroup of a p-divisible group is p-divisible.

Proposition 7. Suppose $f: G \rightarrow G^{\prime}$ is an isomorphism of divisible groups, $A, C$ are multigroups of $G$ and $B, D$ are multigroups of $G^{\prime}$ such that $A \subseteq C$ and $B \subseteq D$. If $A$ and $B$ are pure multigroups of $G$ and $G^{\prime}$, respectively then

(i) $f(A)$ is a pure multigroup of $G^{\prime}$,

(ii) $f^{-1}(B)$ is a pure multigroup of $G$.

Proof. Let $x, y \in G$ and $w, z \in G^{\prime}$ such that $f(x, y)=w, z$. Since $G, G^{\prime}$ are divisible groups for every $n \in \mathbb{N}$, $\exists y \in G$ and $z \in G^{\prime}$ such that $n y=x, n z=w$. From Proposition 2, it follows that $f(A)$ and $f^{-1}(B)$ are multigroups of $G$ and $G^{\prime}$, respectively. Assume that $C$ and $D$ are constant, then by Theorems 3 and 4 , we deduce that, for any $w \in G^{\prime}$

$$
\begin{aligned}
C_{f(n A)}(w) & =C_{n f(A)}(w)=C_{f(A)}(w) \wedge C_{n f(C)}(w) \\
& =C_{f(A)}(w) \wedge \bigvee_{w=n z} C_{f(C)}(z) \\
& =C_{f(A)}(w)
\end{aligned}
$$


and

$$
\begin{aligned}
C_{f^{-1}(n B)}(x) & =C_{n f^{-1}(B)}(x)=C_{f^{-1}(B)}(x) \wedge C_{n f^{-1}(D)}(x) \\
& =C_{f^{-1}(B)}(x) \wedge \bigvee_{x=n y} C_{f^{-1}(D)}(y) \\
& =C_{f^{-1}(B)}(x) .
\end{aligned}
$$

Hence, the results.

Theorem 11. Let $\left\{A_{i}\right\}_{i \in I}$ and $\left\{B_{j}\right\}_{j \in J}$ be families of multigroups of $G$ such that $\left\{A_{i}\right\}_{i \in I} \subseteq\left\{B_{j}\right\}_{j \in J}$ and $\left\{B_{j}\right\}_{j \in J}$ is constant. If $\left\{A_{i}\right\}_{i \in I}$ is pure, then $\bigcap_{i \in I} A_{i}$ is a pure multigroup of $G$.

Proof. Certainly, $\bigcap_{i \in I} A_{i}$ is a multigroup of $G$ by Proposition 1 . If every $A_{i}$ is pure, then suppose $x \in n G$, we have

$$
\begin{aligned}
C_{n\left(\bigcap_{i \in I} A_{i}\right)}(x) & =C_{\left(\bigcap_{i \in I} A_{i}\right) \cap n\left(\bigcap_{j \in J} B_{j}\right)}(x) \\
& =\bigwedge_{i \in I} C M_{A_{i}}(x) \wedge \bigvee_{x=n y} \bigwedge_{j \in J} C_{B_{j}}(y) \\
& =\bigwedge_{i \in I} C_{A_{i}}(x) \wedge \bigwedge_{j \in J} \bigvee_{x=n y} C_{B_{j}}(y) \\
& =\bigwedge_{i \in I} C_{A_{i}}(x)=C_{\bigcap_{i \in I} A_{i}}(x) .
\end{aligned}
$$

Again, suppose $x \notin n G$, then

$$
C_{n\left(\bigcap_{i \in I} A_{i}\right)}(x)=C_{\left(\bigcap_{i \in I} A_{i}\right) \cap n\left(\bigcap_{j \in J} B_{j}\right)}(x)=0=C_{\bigcap_{i \in I} A_{i}}(x) .
$$

Hence, $\bigcap_{i \in I} A_{i}$ is a pure multigroup of $G$.

Theorem 12. Let $\left\{A_{i}\right\}_{i \in I}$ and $\left\{B_{j}\right\}_{j \in J}$ be families of multigroups of $G$ such that $\left\{B_{j}\right\}_{j \in J}$ is constant and contains $\left\{A_{i}\right\}_{i \in I}$. If $\left\{A_{i}\right\}_{i \in I}$ is pure, then

(i) $\bigcup_{i \in I} A_{i}$ is a pure multigroup of $G$ for $A_{1} \subseteq A_{2} \subseteq \ldots \subseteq A_{n}, i=1, \ldots, n$.

(ii) $\Sigma_{i \in I} A_{i}$ is a pure multigroup of $G$.

Proof. By synthesizing Proposition 1 and following the same argument in Theorem 11, the proofs of (i) and (ii) are established.

Theorem 13. Suppose $A$ and $B$ are multigroups of $G$ such that $A \subseteq B$ and $B$ is constant. Then $A$ is pure if and only if $A$ is a divisible multigroup of $G$.

Proof. Suppose $A$ is a pure multigroup of $G$. Then $n A=A \cap n B$ for $n \in \mathbb{N}$. Certainly, $A \cap n B=A$ because

$$
\begin{aligned}
C_{A}(x) \wedge C_{n B}(x) & =C_{A}(x) \wedge \bigvee_{n y=x} C_{B}(y) \\
& =C_{A}(x) \forall x \in X
\end{aligned}
$$

Thus, $n A=A$ for $n \in \mathbb{N}$, and hence $A$ is a divisible multigroup of $G$.

Conversely, assume $A$ is a divisible multigroup of $G$. Then $n A=A$ for $n \in \mathbb{N}$. Since $A \cap n B=A$, we have $n A=A \cap n B n \in \mathbb{N}$, and so $A$ is a pure multigroup of $G$.

Proposition 8. Suppose $A$ and $B$ be multigroups of $G$ such that $A \subseteq B$ and $B$ is constant. Then $A$ is pure if and only if it is p-pure for every prime $p$.

Proof. Similar to Proposition 6. 
Proposition 9. Let $A$ and $B$ be multigroups of $G$ such that $A \subseteq B$ and $B$ is constant. If $A$ is a pure multigroup of $G$, then $A_{n}, n \in \mathbb{N}$ is a pure subgroup of $G$ for $n \leq C_{A}(0)$ and $A^{n}, n \in \mathbb{N}$ is a pure subgroup of $G$ for $n \geq C_{A}(0)$.

Proof. Similar to Theorem 5.

Proposition 10. Suppose $A$ and $B$ are multisets of $G$ bounded by $G^{n}$ such that $A \subseteq B$ and $B$ is constant. If $C_{A}(0)=n$ and every $A_{n}, n \in \mathbb{N}$, is a pure subgroup of $G$, then $A$ is a pure multigroup of $G$.

Proof. Similar to Theorem 6.

Corollary 14. Let $A$ and $B$ be multisets of $G$ bounded by $G^{n}$ such that $A \subseteq B$ and $B$ is constant. If $C_{A}(0)=n$ and every $A^{n}, n \in \mathbb{N}$, is a pure subgroup of $G$, then $A$ is a pure multigroup of $G$.

Proof. Similar to Corollary 7.

\section{Conclusion}

Multigroup theory is an application of group theory to multisets. Numerous analog ideas of group theory have been extended to multigroups. In this paper, we have introduced the concepts of divisible and pure multigroups with a number of some related results. The interconnection between divisible and pure multigroups and that of divisible and pure groups were established with the aid of multigroup cuts. Additional characterizations of divisible and pure fuzzy multigroups could be explored in future research.

Acknowledgments: "The authors would like to thank the referee for improving the readability of the paper."

Author Contributions: All authors contributed equally to the writing of this paper. All authors read and approved the final manuscript.

Conflicts of Interest: "The authors declare no conflict of interest."

\section{References}

[1] Knuth, D. (1981). The art of computer programming. Semi Numerical Algorithms, Second Edition, volume 2, Addison-Wesley, Reading, Massachusetts.

[2] De Bruijin, N. G. (1983). Denumerations of rooted trees and multisets. Discrete Applied Mathematics, 6, 25-33.

[3] Blizard, W. D. (1989). Multiset theory. Notre Dame Journal of Formal Logic, 31, 36-65.

[4] Blizard, W. D. (1991). The development of multiset theory. Modern Logic, 1(4), 319-352.

[5] Girish, K. P. \& John, S. J. (2009). Relations and functions in multiset context. Information Sciences, 179, 758-768.

[6] Jena, S. P., Ghosh, S. K. \& Tripathy, B. K. (2001). On the theory of bags and lists. Information Sciences, 132, 241-254.

[7] Singh, D., Ibrahim, A. M., Yohanna, T. \& Singh, J. N. (2007). An overview of the applications of multisets. Novi Sad Journal of Mathematics, 37(2), 73-92.

[8] Syropoulos, A. (2000, August). Mathematics of multisets. In Workshop on Membrane Computing (pp. 347-358). Springer, Berlin, Heidelberg.

[9] Nazmul, Sk., Majumdar, P. \& Samanta, S. K. (2013). On multisets and multigroups. Annals of Fuzzy Mathematics and Informatics, 6(3), 643-656.

[10] Ejegwa, P. A. (2018). A study of multigroup structure and its acting principles on multiset. A PhD Thesis, Ahmadu Bello University, Zaria, Nigeria.

[11] Awolola, J. A. \& Ibrahim, A. M. (2016). Some results on multigroups. Quasigroups And Related Systems, 24(2), 169-177.

[12] Ejegwa, P. A. \& Ibrahim, A. M. (2017). Some homomorphic properties of multigroups. Buletinul Academiei de Stiinte a Republicii Moldova. Matematica, 83(1), 67-76.

[13] Ejegwa, P. A. (2017). Upper and lower cuts of multigroups. Prajna International Journal of Mathematical Sciences and Applications, 1(1), 19-26.

[14] Ejegwa, P. A. \& Ibrahim, A. M. (2018). Homomorphism of cuts of multigroups. Gulf Journal of Mathematics, 6(1), 61-73.

[15] Awolola, J. A. (2019). On multiset relations and factor multigroups. Journal South East Asian Journal of Mathematics and Mathematical Sciences, 15(3), 1-10.

[16] Awolola, J. A. (2019). On cyclic multigroup family. Ratio Mathematica, 37, 61-68.

[17] Awolola, J. A. \& Ejegwa, P. A. (2017). On some algebraic properties of order of an element of a multigroup. Quasigroups And Related Systems, 25(1), 21-26.

[18] Ejegwa, P. A. (2020). Concept of anti multigroups and its properties. Earthline Journal of Mathematical Sciences, 4(1), 83-97. 
[19] Ejegwa, P. A. \& Ibrahim, A. M. (2017). Normal submultigroups and comultisets of a multigroup. Quasigroups And Related Systems, 25(2), 231-244.

[20] Ejegwa, P. A. \& Ibrahim, A. M. (2017). Direct product of multigroups and its generalization. International Journal of Mathematical Combinatorics, 4, 1-18.

[21] Ejegwa, P. A. \& Ibrahim, A. M. (2017). On comultisets and factor multigroups. Theory and Applications of Mathematics $\mathcal{E}$ Computer Science, 7(2), 124-140.

[22] Ejegwa, P. A. \& Ibrahim, A. M. (2019). Some group's analogous results in multigroup setting. Annals of Fuzzy Mathematics and Informatics, 17(3), 231-245.

[23] Ejegwa, P. A. \& Ibrahim, A. M. (2020). Some properties of multigroups. Palestine Journal of Mathematics, 9(1), 31-47.

[24] Ibrahim, A. M. \& Ejegwa, P. A. (2017). Multigroup actions on multiset. Annals of Fuzzy Mathematics and Informatics, $14(5), 515-526$.

[25] Ibrahim, A. M. \& Ejegwa, P. A. (2017). Characteristic submultigroups of a multigroup. Gulf Journal of Mathematics, $5(4), 1-8$.

[26] Suma, P. \& John, S. J. (2020). Multiset approach to algebraic structures: In Handbook of Research on Emerging Applications of Fuzzy Algebraic Structures, IGI Global Publisher, Hershey, Pennsylvania 17033-1240, USA, 78-90.

[27] Tripathy, B. C., Debnath, S. \& Rakshit, D. (2018). On multiset group. Proyecciones Journal of Mathematics, 37(3), 479-489.

[28] Ejegwa, P. A. \& Agbetayo, J. M. (2020). Some results on commutators in multigroup framework. Journal of the Ramanujan Mathematical Society, 7(2), 67-82.

[29] Debnath, S. \& Debnath, A. (2019). Study of ring structure from multiset context. Applied Sciences, 21, 84-90.

[30] Sidky, F. I. \& Mishref, M. A. (1990). Divisible and pure fuzzy subgroups. Fuzzy Sets and Systems, 34, $377-382$.

[31] Wenxiang, G. \& Tu, L. (1993). The properties of fuzzy divisible groups, Fuzzy Sets and Systems, 56, 195-198.

[32] Yamak,S., Kazanc, O. \& Davvaz, B. (2008). Divisible and pure intuitionistic fuzzy subgroups and their properties. International Journal of Fuzzy Systems, 10(4), 298-307.

(C) 2020 by the authors; licensee PSRP, Lahore, Pakistan. This article is an open access article distributed under the terms and conditions of the Creative Commons Attribution (CC-BY) license (http://creativecommons.org/licenses/by/4.0/). 